CLNS-95/1348

hep-th/9507012

June 1995

\title{
Enhanced Gauge Symmetries and K3 Surfaces
}

\author{
Paul S. Aspinwall \\ F.R. Newman Lab. of Nuclear Studies, \\ Cornell University, \\ Ithaca, NY 14853
}

\begin{abstract}
String-string duality dictates that type IIA strings compactified on a K3 surface acquire non-abelian gauge groups for certain values of the K3 moduli. We argue that, contrary to expectation, the theories for which such enhanced gauge symmetries appear are not orbifolds in the string sense. For a specific example we show that a theory with enhanced gauge symmetry and an orbifold theory have the same classical K3 surface as a target space but the value of the "B-field" differs. This raises the possibility that the conformal field theory associated to a string theory with an enhanced gauge group is badly behaved in some way.
\end{abstract}




\section{Introduction}

There is now strong evidence that the type IIA superstring compactified on a K3 surface is equivalent to the heterotic string compactified on a 4-torus [1], 2, 3, 4, 5, 6]. Both theories give a theory of non-chiral $N=2$ supergravity in 6 dimensions and the moduli spaces are identical. At a generic point in this moduli space the gauge group in both theories is $\mathcal{G} \cong U(1)^{24}$. The manifest difference in the world-sheet point of view of these theories is explained by saying that the strong string-coupling limit of one is the weak string-coupling limit of the other.

In the case of the heterotic string compactified on the 4-torus, it is known that the gauge group in general can be $\mathcal{G} \cong \mathcal{G}_{0} \times U(1)^{4}$, where $\mathcal{G}_{0}$ contains a semi-simple simply-laced Lie group. This occurs for special points in the moduli space where some vector states become massless. An interesting question is what the corresponding statement for the type IIA string is. It is known that from a world-sheet point of view, the gauge group must always be abelian. Thus if this string-string duality is to work, the extra massless vector particles in the type II theory must come from non-perturbative effects, i.e., they are solitons.

Classically, as we will review below, one can show that the K3 surfaces giving such enhanced gauge symmetries for type II strings have quotient singularities and are thus orbifolds as far as target space geometry is concerned [4. It will be important to understand what is meant by the term "orbifold" and we will state it here. Geometrically, an orbifold is a space whose only singularities are locally in the form of some smooth space (usually $\mathbb{C}^{n}$ ) modded out by some discrete group fixing the origin. A special case of such an orbifold is the case of a global orbifold which may be written in the form $Y / K$ for some smooth manifold, $Y$, admitting some group of automorphisms, $K$. In this global case, one can also define an orbifold from a string theory, or conformal field theory, point of view [7] by directly taking the quotient of the field theory describing $Y$ by the group $K$. It is well-known that such orbifold theories are well-behaved despite the apparent target space being singular. Thanks to quantum geometry there is a subtle difference between geometrical and string orbifolds which we will explain later. It is probable that general features of string orbifolds can also be extended to the case where the target space is only locally of the form of a quotient [8] so that it should be of little importance whether an orbifold is global or not. In this letter, any specific examples of orbifolds explicitly described will be of the global type.

It is natural to speculate that the theories with enhanced gauge symmetry are orbifolds from the conformal field theory point of view as well as being orbifolds from the geometrical point of view. The conformal field theory view of an orbifold is a perfectly well-behaved theory and thus this enhancement of the gauge group would correspond to solitons becoming massless when the underlying two-dimensional point of view is smooth. This leads to surprising conclusions as discussed in [4]. This scenario would tell us, for example, that we cannot trust the results of conformal field theory to give us the correct massless spectrum even when the conformal field theory is well-behaved. This should also be contrasted with 
the recent work of [9] where solitons become massless at points in the moduli space where the conformal field theory is singular.

We will show in this letter that, in a least one case, this classical reasoning does not work and the point in moduli space representing the conformal field theory orbifold and the point representing the theory with enhanced gauge symmetry do not coincide - they have a different value of the " $B$-field" which cannot be observed using classical geometry since it is the component of the $B$-field which is hidden away in the quotient singularity. We believe this will be a general phenomenon. The fact that the point of enhanced gauge symmetry is not a string orbifold leaves open the possibility that the conformal field theory description of this point is badly-behaved in some way.

In section 2 we will analyze the enhanced gauge groups from the heterotic string point of view. This allows us in section 3 to describe these points in terms of type II strings in K3 surfaces. We then use discrete symmetries in section 1 to give an example of an orbifold which cannot have any enhanced gauge group.

\section{The Heterotic String}

Let us first pin-point in the moduli space of heterotic string theories where the enhanced gauge groups arise. The moduli space is of the form [10]

$$
\mathscr{M}_{\mathrm{H}} \cong \operatorname{Gr}\left(\Pi, \mathbb{R}^{4,20}\right) / O\left(\Lambda^{4,20}\right),
$$

where $\Pi$ is a space-like 4-plane and $\operatorname{Gr}\left(\Pi, \mathbb{R}^{4,20}\right)$ denotes the Grassmanian of such planes passing through the origin of $\mathbb{R}^{4,20}$ (often written $O(4,20) /(O(4) \times O(20))$ ). The lattice $\Lambda^{4,20}$ is an even self-dual lattice of rank 24 embedded in $\mathbb{R}^{4,20}$ and thus of the form $\left(E_{8}\right)^{\oplus 2} \oplus H^{\oplus 4}$, where $E_{8}$ is minus the Cartan matrix of the Lie algebra $E_{8}$ and

$$
H=\left(\begin{array}{ll}
0 & 1 \\
1 & 0
\end{array}\right)
$$

The group $O\left(\Lambda^{4,20}\right)$ is the automorphism group of this lattice (often written $O(4,20 ; \mathbb{Z})$ ).

The lattice $\Pi \cap \Lambda^{4,20}$ represents the set of right-moving winding/momenta modes of the compactified heterotic string (before the GSO projection) and the lattice $\Pi^{\perp} \cap \Lambda^{4,20}$ represents the corresponding set of left-moving states. Consider an element $\alpha \in \Pi^{\perp} \cap \Lambda^{4,20}$ such that

$$
\alpha^{2}=-2 \text {. }
$$

We will say that such an element has "length" equal to -2 . Such a state will be massless in the six-dimensional theory. In fact, it will be a charged vector and it is not difficult to show that the set of elements of $\alpha \in \Pi^{\perp} \cap \Lambda^{4,20}$ satisfying (3) will form the set of roots for the semi-simple part of the gauge group $\mathcal{G}_{0}$. Note that supersymmetry kills the corresponding statement in the right-moving sector.

Thus we see that any simply-laced algebra that can be embedded in this lattice can be realized by tilting $\Pi$ to be orthogonal to this sub-lattice generated by the simple roots. 


\section{Strings on K3 Surfaces}

Now let us review the form of the moduli space of type IIA strings compactified on a K3 surface. This is equivalent to the moduli space of $N=4$ superconformal field theories with $\mathrm{K} 3$ target space and is thus of the form described in [2]. That is,

$$
\mathscr{M}_{\mathrm{II}} \cong \operatorname{Gr}\left(\Pi, \mathbb{R}^{4,20}\right) / O\left(\Lambda^{4,20}\right),
$$

as required by string-string duality. To interpret this in terms of classical geometry one decomposes $\operatorname{Gr}\left(\Pi, \mathbb{R}^{4,20}\right)$ by the following process.] First choose a primitive null-vector $w \in$ $\Lambda^{4,20}$. Now define $\Sigma^{\prime}=\Pi \cap w^{\perp}$. Then define $B^{\prime}$ as a vector orthogonal to $\Sigma^{\prime}$ such that $B^{\prime} \cdot w=1$ and $\Pi$ is spanned by $\Sigma^{\prime}$ and $B^{\prime}$.

Consider now the subspace of $\mathbb{R}^{4,20}$ given by $w^{\perp} / w$. Such a space is isomorphic to $\mathbb{R}^{3,19}$ and $\Lambda^{4,20} \cap\left(w^{\perp} / w\right)$ is an even self-dual lattice $\Lambda^{3,19}$. This space $w^{\perp} / w$ is taken to represent $H^{2}(\mathrm{~K} 3, \mathbb{R})$ and the sublattice $\Lambda^{3,19}$ gives the lattice of integral cohomology $H^{2}(\mathrm{~K} 3, \mathbb{Z})$. Projecting $\Sigma^{\prime}$ and $B^{\prime}$ into $w^{\perp} / w$ we define $\Sigma$ and $B$ respectively.

The space-like 3-plane $\Sigma \subset H^{2}(\mathrm{~K} 3, \mathbb{R})$ describes a K3 surface, $X$, classically as follows [11]. Let $\Omega$ represent a holomorphic 2 -form on $X$ which is uniquely defined up to scale. It is easy to then show that $\operatorname{Re}(\Omega)$ and $\operatorname{Im}(\Omega)$ span a space-like 2 plane in $H^{2}(X, \mathbb{R})$. If $X$ is taken to have volume one then the Kähler form, $J$, is an element of $H^{2}(X, \mathbb{R})$ satisfying $J^{2}=1 . J$ is also a $(1,1)$-form and is thus orthogonal to $\operatorname{Re}(\Omega)$ and $\operatorname{Im}(\Omega)$. Thus $\operatorname{Re}(\Omega), \operatorname{Im}(\Omega)$ and $J$ span a space-like 3 -plane. This plane is given by $\Sigma$ and the Grassmanian of such planes in $\mathbb{R}^{3,19}$ gives precisely the Teichmüller space for Ricci-flat metrics on a K3 surface of volume 1.

The vector $B$ is simple to interpret. It is the " $B$-field", $B \in H^{2}(X, \mathbb{R})$, for the $\sigma$-model. We have effectively used $w$ to give a decomposition

$$
\operatorname{Gr}\left(\Pi, \mathbb{R}^{4,20}\right) \cong \operatorname{Gr}\left(\Pi, \mathbb{R}^{3,19}\right) \times \mathbb{R}^{+} \times \mathbb{R}^{3,19}
$$

This reads as "factorizing" the moduli space of type IIA strings on a K3 surface into moduli of volume one K3 surfaces, the volume of the K3 surface and the value of the $B$-field respectively. The subgroup of $O\left(\Lambda^{4,20}\right)$ which preserves $w$ may act on the right of equation (5) preserving the decomposition. The subgroup $O\left(\Lambda^{3,19}\right)$ acts on the first factor, as required classically, and the subgroup of translations by $H^{2}(X, \mathbb{Z})$ acts on the last factor as expected from the nonlinear $\sigma$-model.

Thus, given a point in the moduli space $\mathscr{M}_{\mathrm{II}}$ and a null vector $w$, we may determine precisely which K3 surface with which $B$-field our string theory is describing. Note that different choices of $w$ can lead to different geometrical interpretations allowing for stringy equivalences between different K3 surfaces.

\footnotetext{
${ }^{1}$ This construction was made in collaboration with D. Morrison and was used in [2] although it was not explicitly described there.
} 
Now, given the results of section 2, let us determine which K3 surfaces should have enhanced gauge symmetries. Let $L_{\mathcal{G}}$ be the lattice generated by the simple roots of the gauge group. Then we have

$$
L_{\mathcal{G}} \cong \Pi^{\perp} \cap \Lambda^{4,20} .
$$

Let us assume that we may choose $w$ such that $L_{\mathcal{G}}$ lies within $w^{\perp} / w$ (which implies that $\left.\operatorname{rank} L_{\mathcal{G}} \leq 19\right)$. Since the elements of $L_{\mathcal{G}}$ are orthogonal to $\Pi$, they are orthogonal to $\Sigma^{\prime}$. There is a linear function $\phi: \Sigma \rightarrow \mathbb{R}$ such that $x+\phi(x) w \in \Sigma^{\prime}$ for every $x \in \Sigma$. Therefore, the elements of $L_{\mathcal{G}}$ are orthogonal to $\Sigma$.

The elements of $L_{\mathcal{G}}$ are elements of $H^{2}(X, \mathbb{Z})$ are are thus dual to integral 2-cycles. Since they are orthogonal to $\Sigma$ they are orthogonal to $\operatorname{Re}(\Omega)$ and $\operatorname{Im}(\Omega)$ and are therefore $(1,1)$ forms. Thus means that they are dual to algebraic curves in $X$. For the elements of $L_{\mathcal{G}}$ with length -2 , these must be rational curves. The area of these curves is given by the dot product with $J$ which is zero since $J$ lies in $\Sigma$. Thus the simple roots of $\mathcal{G}_{0}$ give rational curves of zero size in $X$. That is, $X$ is a blow-down of some smooth K3 surface $\bar{X}$. The singularities of $X$ are thus locally of the form of a quotient, i.e., $X$ is an orbifold as far as geometry is concerned. This agrees with the expectation that the non-linear $\sigma$-model must break down (due to singularities in the target space) in order for non-perturbative effects to produce an enhanced gauge group. It also nicely ties together the A-D-E classification of surface quotient singularities with the possible simply-laced Lie groups that can appear as gauge groups [1].

The analysis of [4] is not quite complete for our purposes however. It is important to note that the condition for an enhanced gauge symmetry also puts constraints on the $B$-field. For $\Pi$ to be orthogonal to any element $\alpha \in L_{\mathcal{G}}$, it is easy to show from the above that $B . \alpha=0$. That is, the $B$-field must be zero along certain directions. It follows that we may take $X$ to be an orbifold and yet choose $B$ such that there is no enhanced gauge symmetry by taking the values of $B$ to be nonzero in certain directions.

One might expect that the theories for which there is an enhanced gauge symmetry are orbifolds from an conformal field theory point of view as well as being orbifolds from the geometrical point of view. This makes a statement about the value of $B$, as we now explain, that we will show is not correct in at least one example.

Suppose we consider the familiar orbifold $T^{4} / \mathbb{Z}_{2}$. The $\mathbb{Z}_{2}$-action has 16 fixed points resulting in 16 quotient singularities in the orbifold. The orbifold may be blown-up to give a K3 surface. Consider this model from the conformal field theory point of view. We may divide the conformal field theory corresponding to $T^{4}$ by the $\mathbb{Z}_{2}$ symmetry to obtain another conformal field theory which is an orbifold. It is well-known that this orbifold theory is wellbehaved even though its apparent target space $T^{4} / \mathbb{Z}_{2}$ is singular. Now consider the $B$-field degree of freedom. A K3 surface has $b_{2}=22$ and so the $B$-field lives in a 22-dimensional space. The 4 -torus has only 6 independent two-forms and thus the $B$-field in this case lives in only a 6 -dimensional space. Thus, when we make the orbifold in the conformal field theory language we must be implicitly assigning a value to the 16 components of the $B$-field coming 
from the twisted sector. Only if this value is zero can we really assert that our orbifold has an enhanced gauge group.

The value of the $B$-field for an orbifold was studied in [12, 8] for the case of nonlinear $\sigma$-models on complex threefolds. There it was found that $B_{i}=\frac{1}{2}$ for the twisted component of the $B$-field. If we can make some kind of similar statement for the case of complex surfaces we will have shown that conformal field theory orbifolds never have enhanced gauge groups. Actually, the case of complex surfaces is rather harder than the threefold case. In the latter, it was the way that the $B$-field appeared in the instanton expansion of the correlation functions of the chiral primary fields that allowed it to be studied. In the case when the target space has complex dimension two it is simple to show that such instanton effects are trivial. Such effects can be expressed in terms of a topological field theory where the instantons are rational curves [13]. In the case of complex dimension two the target space has a quaternionic structure allowing any of an $S^{2}$ of complex structures to be chosen for a given Ricci-flat metric. Whether a rational curve exists or not depends on this choice of complex structure. Since the field theory cares only about the Ricci-flat metric and not the complex structure it would lead to a contradiction if there were any nonzero instanton

effects. We therefore need some other way to determine $B$. This will be the use of discrete symmetries as we now discuss in the following section.

\section{$4 \quad$ K3 Surfaces with Symmetries}

Consider a K3 surface $X$, with a finite group, $G$, of automorphisms which preserve the holomorphic 2-form, $\Omega$. Such symmetry groups, $G$, are well-understood thanks to the beautiful work of Nikulin [14] for the abelian case and Mukai [15] in the general case.

The general question we wish to address is whether we can find an example of a K3 surface with such a large symmetry group that its position in the moduli space is uniquely determined. Let us first analyze the classical case. To do this we introduce 4 sublattices of $H^{2}(X, \mathbb{Z}) \cong \Lambda^{3,19}$ :

1. $S_{X}$ is the lattice of algebraic cycles, i.e., elements of $H^{2}(X, \mathbb{Z})$ orthogonal to $\operatorname{Re}(\Omega)$ and $\operatorname{Im}(\Omega)$.

2. $T_{X}$ is the lattice of transcendental cycles, i.e., $T_{X}=S_{X}^{\perp} \subset H^{2}(X, \mathbb{Z})$.

3. $T_{X, G}$ is the lattice of $G$-invariant elements of $H^{2}(X, \mathbb{Z})$.

4. $S_{X, G}=T_{X, G}^{\perp} \subset H^{2}(X, \mathbb{Z})$.

We then have the following Lemma (4.2 of [14]):

a) $S_{X, G}$ is nondegenerate and negative definite. 
b) $S_{X, G}$ does not contain any elements, $\alpha$, such that $\alpha^{2}=-2$.

c) $T_{X} \subset T_{X, G}$ and $S_{X, G} \subset S_{X}$.

If we want the automorphism to preserve the Kähler form, $J$, as well as the holomorphic 2 -form we require that the 3-plane $\Sigma$ spanned by $J$ and $\Omega$ lies in $T_{X, G} \otimes \mathbb{R}$. Thus the rank of $S_{X, G}$ can be no larger than 19. If it is 19 then $\Sigma$ is fixed uniquely by $G$.

In [15] the space of total rational cohomology was introduced, $V=H^{*}(X, \mathbb{Q})$. The quantity $\mu(G)$ was introduced, where

$$
\mu(g)=\frac{24}{n \prod_{p \mid n}\left(1+\frac{1}{p}\right)},
$$

where $g \in G, n$ is the order of $g$, the product runs over the prime factors of $n$ and

$$
\mu(G)=\frac{1}{|G|} \sum_{g \in G} \mu(g) .
$$

One may then show that $\mu(G)$ is equal to the dimension of the $G$-invariant part of $V$. Since $H^{0}$ and $H^{4}$ are clearly preserved by $G$ we have that $\mu(G) \geq 5$ with equality fixing $\Sigma$ uniquely.

Consider the case where $X$ is the "Fermat" quartic K3 surface defined as the hypersurface

$$
x_{0}^{4}+x_{1}^{4}+x_{2}^{4}+x_{3}^{4}=0 \quad \subset \mathbb{P}^{3} .
$$

This has symmetries generated by multiplying the homogeneous coordinates, $x_{j}$, by $i$ and by permuting the homogeneous coordinates. All told, we have $G \cong\left(\left(\mathbb{Z}_{4}\right)^{3} \rtimes \mathcal{S}_{4}\right) / \mathbb{Z}_{4}$, where $\mathcal{S}_{4}$ is the symmetric group on 4 elements. An elementary calculation shows that $\mu(G)=5$ and so we have an example where the symmetry is maximal in that $S_{X, G}$ is rank 19 and $\Sigma$ is uniquely fixed.

Let us note at this point the significance of the fact that $S_{X, G}$ contains no elements of length -2 . From our discussion in section 3 we see that, since $\Sigma$ is orthogonal to elements in $S_{X, G}$, an element of length -2 would give a quotient singularity in $X$. Thus $X$ cannot be an orbifold. In this case it is obvious - the Fermat quartic surface is indeed smooth but we see that having a large symmetry group itself prevents $X$ acquiring singularities. It is this statement that we now wish to extend to the quantum case.

Let us extend the above to the case of stringy geometry along the lines suggested in [2]. Now we take the lattice $H^{*}(X, \mathbb{Z}) \cong \Lambda^{4,20}$ and allow $G$ to act on that. We define lattices in analogy with above, namely let $\widehat{T}_{X, G}$ be the sublattice of $H^{*}(X, \mathbb{Z})$ preserved by $G$, and $\widehat{S}_{X, G}$ be the orthogonal complement in $H^{*}(X, \mathbb{Z})$. The analogy of the statement that $G$ preserves $\Omega$ in the quantum case is that $G$ preserves the chiral primary field of charge $(2,0)$ of the conformal field theory. In this case $\Pi \subset \widehat{T}_{X, G} \otimes \mathbb{R}$. Thus $\widehat{S}_{X, G}$ has rank $\leq 20$. 
This may be related to the classical case as follows. Choose a primitive null vector $w$ as in section 3 to fix the geometrical interpretation of the theory. We then claim that $G_{c}$, the group of classical symmetries, is the subgroup of $G$ that fixes $w$ and that

$$
S_{X, G_{c}}=\widehat{S}_{X, G} \cap w^{\perp} / w
$$

This means that by choosing various w's we may extend Nikulin's lemma to the quantum

case. In particular $\widehat{S}_{X, G}$ is negative definite and has no elements of length -2 .

If we can find an example where $\widehat{S}_{X, G}$ is of rank 20 then this will fix $\Pi$ uniquely. We can do this by taking a case where the classical K3 surface has rank $S_{X, G_{c}}=19$ and there is a "quantum symmetry" present in addition. A quantum symmetry is a symmetry of the string theory but not of the classical geometry and so must be an element of $G$ that does not preserve $w$.

An example of this is found by taking the Gepner model [16] of the Fermat quartic K3 surface. This model amounts to taking a particular value for the Kähler form and $B$-field and it has a non-trivial quantum symmetry as described in [17]. (The extra $\mathbb{Z}_{4}$ which appears at this point does not itself preserve the $(2,0)$-field but one can mix this with other classical symmetries present to produce one that does.) It follows that the quartic K3 surface at the Gepner point is a model with rank $\widehat{S}_{X, G}=20$. In particular, since $\Pi^{\perp} \cap \Lambda^{4,20}$ is $\widehat{S}_{X, G}$ and so has no elements of length -2 , by string-string duality we infer that this model has no enhanced gauge symmetry.

It now remains to show that this model has an orbifold interpretation. This is actually quite easy given the Greene-Plesser mirror construction [18]. The mirror of this quartic K3 surface, $X$ by this construction is the orbifold $X /\left(\mathbb{Z}_{4} \times \mathbb{Z}_{4}\right)$. Type IIA strings compactified on a K3 surface are self-mirror [19 and so this orbifold theory is just a different geometrical interpretation of the same string theory — we have just chosen another $w$. Thus we have given an example of an orbifold conformal field theory with no enhanced gauge symmetry. This means that the effective value of the $B$-field is nonzero along certain twisted directions. Analyzing the fixed-point set of this orbifold one sees that the gauge group will be $\mathcal{G} \cong$ $S U(4)^{6} \times U(1)^{6}$ if we move a finite distance in the moduli space by integrating along the twisted marginal operators that will take the value of the $B$-field to zero.

\section{Conclusions}

Classically, for the case of type IIA strings on a K3 surface, the enhanced gauge groups appear when the underlying space is an orbifold but we have shown here that quantum geometry can move this point away from the conformal field theory orbifold point in the moduli space of string theories. That is, we have given an example of an orbifold in the string (or conformal field theory) sense which has no enhanced gauge symmetry. Suppose, for a moment, that we could find a string orbifold which did have an enhanced gauge group when used to compactify 
the type IIA string. This would mean that the conformal field theory approach was unable to compute the massless spectrum of states despite being apparently well-behaved. This is to be contrasted with the situation in [9] where massless solitons coincided with badly behaved conformal field theories. It would be interesting to see if the conformal field theory associated with the enhanced gauge symmetry point is bad in some way. Indeed, in section 5.5 of [12] an example was given in the threefold case of an orbifold theory which becomes singular when the $B$-field is taken to zero. If one can understand such effects in the case of two complex dimensions, this may provide a method of generalizing the results of this letter.

\section{Acknowledgements}

It is a pleasure to thank M. Gross, D. Morrison, S. Shenker, H. Tye and E. Witten for useful conversations. The work of the author is supported by a grant from the National Science Foundation.

\section{References}

[1] N. Seiberg, Observations on the Moduli Space of Superconformal Field Theories, Nucl. Phys. B303 (1988) 286-304.

[2] P. S. Aspinwall and D. R. Morrison, String Theory on K3 Surfaces, Duke and IAS 1994 preprint DUK-TH-94-68, IASSNS-HEP-94/23, hep-th/9404151, to appear in "Essays on Mirror Manifolds 2".

[3] C. Hull and P. Townsend, Unity of Superstring Dualities, Nucl. Phys. B438 (1995) 109-137.

[4] E. Witten, String Theory Dynamics in Various Dimensions, IAS 1995 preprint IASSNSHEP-95-18, hep-th/9503124.

[5] J. Harvey and A. Strominger, The Heterotic String is a Soliton, EFI and UCSB 1995 preprint EFI-95-16, hep-th/9504047.

[6] A. Sen, String String Duality Conjecture in Six Dimensions and Charged Soliton Strings, Tata 1995 preprint TIFR-TH-95-16, hep-th/9504027.

[7] L. Dixon, J. A. Harvey, C. Vafa, and E. Witten, Strings on Orbifolds, Nucl. Phys. B261 (1985) 678-686, and B274 (1986) 285-314.

[8] P. S. Aspinwall, Resolution of Orbifold Singularities in String Theory, IAS 1994 preprint IASSNS-HEP-94/9, hep-th/9403123, to appear in "Essays on Mirror Manifolds 2". 
[9] A. Strominger, Massless Black Holes and Conifolds in String Theory, UCSB 1995 preprint, hep-th/9504090.

[10] K. S. Narain, New Heterotic String Theories in Uncompactified Dimensions < 10, Phys. Lett. 169B (1986) 41-46.

[11] A. L. Besse, Einstein Manifolds, Springer-Verlag, Berlin, 1987.

[12] P. S. Aspinwall, B. R. Greene, and D. R. Morrison, Measuring Small Distances in N=2 Sigma Models, Nucl. Phys. B420 (1994) 184-242.

[13] E. Witten, Mirror Manifolds and Topological Field Theory, in S.-T. Yau, editor, "Essays on Mirror Manifolds", International Press, 1992.

[14] V. V. Nikulin, Finite Automorphism Groups of Kähler K3 Surfaces, Trans. Moscow Math. Soc. 38 (1979) 71-135.

[15] S. Mukai, Finite Groups of Automorphisms of K3 Surfaces and the Mathieu Group, Invent. Math. 94 (1988) 183-221.

[16] D. Gepner, Exactly Solvable String Compactifications on Manifolds of SU(N) Holonomy, Phys. Lett. 199B (1987) 380-388.

[17] C. Vafa, Quantum Symmetries of String Vacua, Mod. Phys. Lett. A4 (1989) 1615-1626.

[18] B. R. Greene and M. R. Plesser, Duality in Calabi-Yau Moduli Space, Nucl. Phys. B338 (1990) 15-37.

[19] P. S. Aspinwall and D. R. Morrison, U-Duality and Integral Structures, Cornell 1995 preprint CLNS-95/1334, hep-th/9505025, to appear in Phys. Lett. B. 